\title{
ON A CELL ENTROPY INEQUALITY FOR DISCONTINUOUS GALERKIN METHODS
}

\author{
GUANGSHAN JIANG AND CHI-WANG SHU
}

\begin{abstract}
We prove a cell entropy inequality for a class of high-order discontinuous Galerkin finite element methods approximating conservation laws, which implies convergence for the one-dimensional scalar convex case.
\end{abstract}

\section{INTRODUCTION}

In [3] Cockburn and Shu defined a class of discontinuous Galerkin finite element methods for conservation laws (the multidimensional case was discussed in [4]):

$$
u_{t}+\operatorname{div} f(u)=0 .
$$

The entropy solution of (1.1) also satisfies

$$
U(u)_{t}+\operatorname{div} F(u) \leq 0
$$

in the distribution sense, for any convex function $U(u)$ and consistent entropy flux $F(u)$ satisfying $F^{\prime}(u)=U^{\prime}(u) f^{\prime}(u)$. The scheme is obtained in the following way: first a triangulation $\mathscr{T}_{h}$ is chosen. In one space dimension, $\mathscr{T}_{h}$ is just a collection of subintervals $I_{j}=\left(x_{j-\frac{1}{2}}, x_{j+\frac{1}{2}}\right)$, which are not necessarily of the same length. We will use the notation $\Delta x_{j}=x_{j+\frac{1}{2}}-x_{j-\frac{1}{2}}, h=\max _{j} \Delta x_{j}$ and $x_{j}=\frac{1}{2}\left(x_{j-\frac{1}{2}}+x_{j+\frac{1}{2}}\right)$. In two and more space dimensions, $\mathscr{T}_{h}$ is a collection of triangles, tetrahedrons or other simple geometric objects. The solution space $V_{h}$ is defined as the collection of all piecewise polynomials of degree up to $r$ for an $(r+1)$ st-order method. The functions in $V_{h}$ are allowed to have discontinuities across element interfaces. The conservation law (1.1) is multiplied by a test function $v \in V_{h}$, integrated over an element $K \in \mathscr{T}_{h}$, and formally integrated by parts to shift the spatial derivatives from $f(u)$ to $v$. The result

Received by the editor June 1, 1993.

1991 Mathematics Subject Classification. Primary 65M60, 65M12, 35L65.

Key words and phrases. Conservation law, discontinuous Galerkin, entropy condition, convergence.

Research supported by ARO Grant DAAL03-91-G-0123, NSF Grant DMS-9211820, NASA Langley Grant NAG-1-1145 and Contract NAS1-19480 while the second author was in residence at ICASE, NASA Langley Research Center, Hampton, VA 23681-0001, and AFOSR Grant 93-0090. 
is:

$$
\begin{aligned}
\int_{K} u_{t}(x, t) v(x) d x & +\sum_{e \in \partial K} \int_{e} f(u(x, t)) \cdot n v(x) d s \\
& -\int_{K} f(u(x, t)) \cdot \operatorname{grad} v(x) d x=0
\end{aligned}
$$

where $\partial K$ is the boundary of the element $K$ and $n$ is the unit outward normal vector. Up to now, this is just the standard discontinuous Galerkin method. Two things from the finite difference methodology are then used in [3], [4]: one is the monotone or $E$-flux (approximate Riemann solver for systems) to define the trace of the physical flux $f(u)$ at the cell interface; the other is a local nonlinear limiter which limits the values of $u$ at the cell interface by the differences of the means of $u$ over elements. With these two ingredients, it was proved in [3] and [4] that the schemes are formally high-order accurate, measured by local truncation errors in smooth regions including at extrema, are total-variation bounded for one space dimension, and are maximum-norm bounded for any space dimensions. The only thing missing is the entropy condition: in [3], we were able to prove entropy consistency for the square entropy $U(u)=\frac{u^{2}}{2}$ for one-dimensional convex $f(u)$ with an $h$-independent modification to the scheme, following the idea of Osher [12]. We were also able to prove entropy consistency for all convex entropies with an $h$-dependent modification to the scheme. The $h$-dependent limiters make the proof of high-order schemes easy, but they are not very desirable for practical computations, because they usually limit the slope near the discontinuities stronger than necessary, and essentially flatten the solution to piecewise constants there for a fine mesh. They also destroy the self-similarity of the scheme.

The entropy condition seems difficult to prove for high-order finite difference schemes. Osher and Tadmor proved [13] that finite difference schemes (those which evolve only the means) which satisfy cell entropy inequalities for all convex entropies can be at most first-order accurate. Even for one entropy inequality (say for the square entropy), for one space dimension and for convex $f(u)$, the proof is extremely elusive if one does not modify the scheme. Osher [11] and Nessyahu and Tadmor [10] were able to get such cell entropy inequalities, for the square entropy and for the second-order MUSCL scheme, with some $h$-independent modifications; Yang [14] was able to prove convergence of the unmodulated second-order MUSCL scheme, using a global analysis rather than relying solely on cell entropy inequalities; Lions and Souganidis [8] proved convergence of the second-order MUSCL scheme for steady-state HamiltonJacobi equations and conservation laws. There are also many results which prove entropy consistency and/or convergence using $h$-dependent limiters or modifications for high-order schemes: for example, Coquel and LeFloch [7] for finite difference; Johnson, Szepessy, and Hansbo [9] for streamline diffusion finite element; Cockburn, Coquel, and LeFloch [5] and Cockburn and Gremaud [6] for high-order finite volume, streamline diffusion or discontinuous Galerkin schemes with $h$-dependent "shock capturing" terms. These results are usually more general (many space dimensions, nonconvex fluxes, etc.). However, as we have indicated before, $h$-dependent limiters or modifications should be 
avoided for practical computations if at all possible. More recently, Bouchut, Bourdarias, and Perthame [2] obtained a second-order one-dimensional scheme which is consistent with all entropy conditions and does not use $h$-dependent limiter. A key ingredient of [2] is to evolve both the mean and the slope and to use the whole function (not just the mean) to obtain cell entropy inequalities. Discontinuous Galerkin methods also fall into this category (evolving the whole polynomial in a cell, rather than just the mean), and we use this fact strongly in this paper.

In $\S 2$ we prove a cell entropy inequality using the square entropy $U(u)=\frac{u^{2}}{2}$ for the unmodulated semidiscrete discontinuous Galerkin method of [3], [4]. The proof is remarkably simple and does not even use any nonlinear limiters. It thus works for any spatial order of accuracy. As far as we know, all the previous cell entropy inequalities without $h$-dependent limiters must restrict the slope of a function by $\frac{1}{\Delta x_{j}} \min \bmod \left(\Delta_{+} u_{j}, \Delta_{-} u_{j}\right)$ (this means that the difference at the interface, $u_{j+\frac{1}{2}}^{+}-u_{j+\frac{1}{2}}^{-}$, must be of the same sign as that of $u_{j+1}-u_{j}$, i.e., no "sawtooth" is allowed in the reconstruction), hence cannot be higher than second-order accurate. The result in this paper illustrates the potential of discontinuous Galerkin methods, or equivalent Hermite-type finite difference/finite volume-type methods, which evolve the whole polynomial in the cell rather than just the mean. Time discretization is discussed in $\S 3$.

\section{Cell ENTROPY INEQUALITY FOR THE SQUARE ENTROPY}

The discontinuous Galerkin scheme in one space dimension, defined in [3], in its semidiscrete form without slope limiting, is the following: Find $u(\cdot, t) \in V_{h}$ such that, for all $v \in V_{h}$ and all subintervals $I_{j}$,

$$
\begin{aligned}
\int_{I_{j}} u_{t}(x, t) v(x) d x+h_{j+\frac{1}{2}}(t) & v\left(x_{j+\frac{1}{2}}^{-}\right)-h_{j-\frac{1}{2}}(t) v\left(x_{j-\frac{1}{2}}^{+}\right) \\
& -\int_{I_{j}} f(u(x, t)) v_{x}(x) d x=0 .
\end{aligned}
$$

Here, $h_{j+\frac{1}{2}}(t)=h\left(u\left(x_{j+\frac{1}{2}}^{-}, t\right), u\left(x_{j+\frac{1}{2}}^{+}, t\right)\right)$ is a Lipschitz continuous monotone flux (i.e., $h$ is nondecreasing in the first argument and nonincreasing in the second argument), or more generally, an $E$-flux as defined by Osher [11]:

$$
\left(h\left(u^{-}, u^{+}\right)-f(u)\right)\left(u^{+}-u^{-}\right) \leq 0
$$

for all $u$ between $u^{-}$and $u^{+}$. Some examples of the commonly used monotone fluxes can be found in, e.g., [3].

If we take $v(x)=u(x, t)$ in $(2.1)$, we get

$$
\begin{aligned}
\int_{I_{j}}\left(\frac{u^{2}(x, t)}{2}\right)_{t} d x+h_{j+\frac{1}{2}}(t) u\left(x_{j+\frac{1}{2}}^{-}, t\right) & -h_{j-\frac{1}{2}}(t) u\left(x_{j-\frac{1}{2}}^{+}, t\right) \\
& -\int_{I_{j}} f(u(x, t)) u_{x}(x, t) d x=0 .
\end{aligned}
$$


We define

$$
g(u)=\int^{u} f(u) d u
$$

and rewrite $(2.3)$ as

$$
\int_{I_{j}}\left(\frac{u^{2}(x, t)}{2}\right)_{t} d x+\hat{F}_{j+\frac{1}{2}}(t)-\hat{F}_{j-\frac{1}{2}}(t)+A_{j}(t)=0,
$$

where

$$
\hat{F}_{j+\frac{1}{2}}(t)=h_{j+\frac{1}{2}}(t) u\left(x_{j+\frac{1}{2}}^{-}, t\right)-g\left(u\left(x_{j+\frac{1}{2}}^{-}, t\right)\right)
$$

is consistent with the entropy flux for the square entropy,

$$
F(u)=\int^{u} f^{\prime}(u) u d u=f(u) u-\int^{u} f(u) d u=f(u) u-g(u),
$$

and

$$
\begin{aligned}
A_{j}(t)= & -h_{j-\frac{1}{2}}(t)\left(u\left(x_{j-\frac{1}{2}}^{+}, t\right)-u\left(x_{j-\frac{1}{2}}^{-}, t\right)\right) \\
& +g\left(u\left(x_{j-\frac{1}{2}}^{+}, t\right)\right)-g\left(u\left(x_{j-\frac{1}{2}}^{-}, t\right)\right) \\
= & -\left(h_{j-\frac{1}{2}}(t)-f(\xi)\right)\left(u\left(x_{j-\frac{1}{2}}^{+}, t\right)-u\left(x_{j-\frac{1}{2}}^{-}, t\right)\right) \geq 0,
\end{aligned}
$$

where we have used the mean value theorem and the definition (2.4) of $g(u)$ in the second equality; $\xi$ is between $u\left(x_{j-\frac{1}{2}}^{-}, t\right)$ and $u\left(x_{j-\frac{1}{2}}^{+}, t\right)$, and the last inequality is due to the property of E-flux (2.2).

We have thus proved the cell entropy inequality

$$
\int_{I_{j}}\left(\frac{u^{2}(x, t)}{2}\right)_{t} d x+\hat{F}_{j+\frac{1}{2}}(t)-\hat{F}_{j-\frac{1}{2}}(t) \leq 0
$$

for the square entropy $U(u)=\frac{u^{2}}{2}$. Notice that we do not need any nonlinear limiting at this stage. However, nonlinear limiting as introduced in [3] and [4] will not destroy this cell entropy inequality (see next section). The cell entropy inequality (2.9) trivially implies $L^{2}$ stability of the scheme (again without even using the nonlinear limiting):

$$
\frac{d}{d t} \int_{-\infty}^{\infty}\left(\frac{u^{2}(x, t)}{2}\right) d x \leq 0
$$

but it is much stronger. For example, if $f(u)$ is convex and we use the nonlinear limiting [3] to obtain a total-variation boundedness for the solution, we will have convergence towards the unique entropy solution.

The same entropy inequality can be obtained for many space dimensions with arbitrary triangulations:

$$
\int_{K}\left(\frac{u^{2}(x, t)}{2}\right)_{t} d x+\sum_{e \in \partial K} \int_{e} F_{e, K}(x, t) d s \leq 0,
$$

where $F_{e, K}$ is consistent with $F \cdot n_{K}$ for the entropy flux $F$ in (1.2) and the outward normal $n_{K}$, and $F_{e, K}=-F_{e, K^{\prime}}$ for the two neighboring elements 
$K \cap K^{\prime}=\{e\}$ (conservation). We omit the details of the derivation since they parallel those for the one-dimensional case.

We have the following two remarks:

1. If we try to do the same estimate for a general convex entropy $U(u)$, we can obtain exactly the same cell entropy inequality modulo an interpolation error term:

$$
-\int_{I_{j}}\left(u(x, t)_{t}+f(u(x, t))_{x}\right)\left(U^{\prime}(u(x, t))-\Pi U^{\prime}(u(x, t))\right) d x,
$$

where $\Pi v$ is a projection into the space $V_{h}$ which interpolates at the two end points of $I_{j}$. This motivates the following $h$-dependent modification of the scheme, which is similar to the "shock-capturing term" added to the streamline diffusion method in Johnson, Szepessy, and Hansbo [9] and in Cockburn and Gremaud [6]:

$$
\begin{aligned}
\int_{I_{j}} & u_{t}(x, t) v(x) d x+h_{j+\frac{1}{2}}(t) v\left(x_{j+\frac{1}{2}}^{-}\right)-h_{j-\frac{1}{2}}(t) v\left(x_{j-\frac{1}{2}}^{+}\right) \\
& -\int_{I_{j}} f(u(x, t)) v_{x}(x) d x \\
& +C h \int_{I_{j}}\left|u_{t}(x, t)+f(u(x, t))_{x}\right| \frac{u_{x}(x, t)}{\left|u_{x}(x, t)\right|} v_{x}(x) d x=0,
\end{aligned}
$$

where $C$ is a suitable positive constant. This, together with an $L_{\infty}$ bound which can be obtained by using nonlinear limiters [3], will give us a cell entropy inequality for arbitrary convex entropy at least for the $r=1$ (secondorder) case, hence convergence for any nonconvex flux $f(u)$ in this case. For general $r$, more $h$-dependent modification is needed. Notice that by adding this modification, the formal order of accuracy of the scheme is not changed: $\left|u_{t}(x, t)+f(u(x, t))_{x}\right|$ is just the local truncation error. A similar argument as in [9] shows that the modification does not destroy convergence towards weak solutions (conservation).

2. The so-called discontinuous Galerkin method can also be recast as a finite difference scheme (Hermite type: where one evolves both the mean and the slope, maybe more). For example, the second-order case is just the following scheme for the mean $\bar{u}_{j}(t)$ and the slope $s_{j}(t)$ where $u(x, t)=\bar{u}_{j}(t)+$ $s_{j}(t)\left(x-x_{j}\right)$ in cell $I_{j}$ (see [3]):

$$
\begin{aligned}
\frac{d}{d t} \bar{u}_{j}(t)= & -\frac{1}{\Delta x_{j}}\left[\hat{f}_{j+\frac{1}{2}}(t)-\hat{f}_{j-\frac{1}{2}}(t)\right], \\
\frac{d}{d t} s_{j}(t)= & -\frac{6}{\Delta x_{j}^{2}}\left[\hat{f}_{j+\frac{1}{2}}(t)+\hat{f}_{j-\frac{1}{2}}(t)\right] \\
& +\frac{12}{\Delta x_{j}^{3}} \int_{I_{j}} f\left(\bar{u}_{j}(t)+s_{j}(t)\left(x-x_{j}\right)\right) d x,
\end{aligned}
$$

where

$$
\hat{f}_{j+\frac{1}{2}}(t)=h\left(\bar{u}_{j}(t)+\frac{\Delta x_{j}}{2} s_{j}(t), \bar{u}_{j+1}(t)-\frac{\Delta x_{j+1}}{2} s_{j+1}(t)\right) .
$$


We have thus in effect proved cell entropy inequalities for such high-order Hermite-type finite difference schemes without using the help of any nonlinear limiting. Of course, to get convergence, one must use the nonlinear limiting to obtain $L_{\infty}$ and/or total-variation bounds.

\section{TIME DisCRETIZATION}

We discretize (2.1) in time by the following class of methods:

$$
\begin{aligned}
\int_{I_{j}} \frac{u^{n+1}(x)-u^{n}(x)}{\Delta t} v(x) d x+h_{j+\frac{1}{2}}^{n+\theta} v\left(x_{j+\frac{1}{2}}^{-}\right)-h_{j-\frac{1}{2}}^{n+\theta} v\left(x_{j-\frac{1}{2}}^{+}\right) \\
-\int_{I_{j}} f\left(u^{n+\theta}(x)\right) v_{x}(x) d x=0,
\end{aligned}
$$

where

$$
\begin{aligned}
u^{n+\theta}(x) & =(1-\theta) u^{n}(x)+\theta u^{n+1}(x), \\
h_{j+\frac{1}{2}}^{n+\theta} & =h\left(u^{n+\theta}\left(x_{j+\frac{1}{2}}^{-}\right), u^{n+\theta}\left(x_{j+\frac{1}{2}}^{+}\right)\right) .
\end{aligned}
$$

For $\theta=0$, this is the Euler forward discretization; for $\theta=1$, it is Euler backward, and for $\theta=\frac{1}{2}$, Crank-Nicolson.

If we take $v(x)=u^{n+\theta}(x)$, we obtain just as before

$$
\int_{I_{j}} \frac{u^{n+1}(x)-u^{n}(x)}{\Delta t} u^{n+\theta}(x) d x+\hat{F}_{j+\frac{1}{2}}^{n+\theta}-\hat{F}_{j-\frac{1}{2}}^{n+\theta} \leq 0,
$$

where

$$
\hat{F}_{j+\frac{1}{2}}^{n+\theta}=h_{j+\frac{1}{2}}^{n+\theta} u^{n+\theta}\left(x_{j+\frac{1}{2}}^{-}\right)-g\left(u^{n+\theta}\left(x_{j+\frac{1}{2}}^{-}\right)\right)
$$

with $g(u)$ defined by (2.4). We can rewrite (3.3) as

$$
\begin{aligned}
& \int_{I_{j}} \frac{\left(u^{n+1}(x)\right)^{2}-\left(u^{n}(x)\right)^{2}}{2 \Delta t} d x+\hat{F}_{j+\frac{1}{2}}^{n+\theta}-\hat{F}_{j-\frac{1}{2}}^{n+\theta} \\
&+\left(\theta-\frac{1}{2}\right) \int_{I_{j}} \frac{\left(u^{n+1}(x)-u^{n}(x)\right)^{2}}{\Delta t} d x \leq 0 .
\end{aligned}
$$

Thus, a sufficient condition to get the cell entropy inequality

$$
\int_{I_{j}} \frac{\left(u^{n+1}(x)\right)^{2}-\left(u^{n}(x)\right)^{2}}{2 \Delta t} d x+\hat{F}_{j+\frac{1}{2}}^{n+\theta}-\hat{F}_{j-\frac{1}{2}}^{n+\theta} \leq 0
$$

is just $\theta \geq \frac{1}{2}$, i.e., implicit schemes from Crank-Nicolson to Backward Euler.

Up to now, we have not considered the nonlinear minmod-type limiters in [3] and [4]. These limiters will render the scheme total-variation stable (in one space dimension) or $L_{\infty}$ stable (in many space dimensions). We have to make sure that these limiters do not destroy the cell entropy inequality (3.6). This turns out to be a simple issue: suppose $\tilde{u}^{n+1}(x)$ is the solution obtained from the scheme (3.1) without limiter, then projected to get the solution at time level $n+1$ by some minmod-type limiter $u^{n+1}(x)=P \tilde{u}^{n+1}(x)$ (this is the procedure 
adopted in [3] and [4]). The above derivation for the unlimited scheme will give (3.6) for $\tilde{u}^{n+1}$ :

$$
\int_{I_{j}} \frac{\left(\tilde{u}^{n+1}(x)\right)^{2}-\left(u^{n}(x)\right)^{2}}{2 \Delta t} d x+\hat{F}_{j+\frac{1}{2}}^{n+\theta}-\hat{F}_{j-\frac{1}{2}}^{n+\theta} \leq 0 ;
$$

hence, a sufficient condition to get the cell entropy inequality (3.6) for the limited (projected) solution $u^{n+1}$ is to require the projection $P$ to satisfy

$$
\int_{I_{j}}(P w(x))^{2} d x \leq \int_{I_{j}}(w(x))^{2} d x
$$

for all polynomials $w(x)$ of degree up to $r$ (recall that $r+1$ is the order of the scheme), i.e., $P$ does not increase the $L_{2}$ norm of any $r$ th-degree polynomial in cell $I_{j}$. Notice that this is the idea used in [2]. An easy way to ensure condition (3.8) is to write $w(x)$ and $P w(x)$ as expansions of (scaled) Legendre polynomials $p_{l}(x)$ as in [3]: $w(x)=\sum_{l=0}^{r} a_{l} p_{l}(x)$ and $P w(x)=\sum_{l=0}^{r} b_{l} p_{l}(x)$. A simple sufficient condition to ensure (3.8) is now just $\left|b_{l}\right| \leq\left|a_{l}\right|$ for all $l$, which is easily checked to be correct for the $P^{1}$ case $(r=1)$ in [3] and also for the general $P^{r}$ case with similar minmod limiters on $a_{l}$ (see also [1] for such limiters).

It is also possible to get cell entropy inequalities for certain explicit time discretizations. For example, with leap-frog time discretization,

$$
\begin{array}{r}
\int_{I_{j}} \frac{u^{n+1}(x)-u^{n-1}(x)}{2 \Delta t} v(x) d x+h_{j+\frac{1}{2}}(t)^{n} v\left(x_{j+\frac{1}{2}}^{-}\right)-h_{j-\frac{1}{2}}(t)^{n} v\left(x_{j-\frac{1}{2}}^{+}\right) \\
-\int_{I_{j}} f\left(u^{n}(x)\right) v_{x}(x) d x=0,
\end{array}
$$

we can obtain, by taking $v(x)=u^{n}(x)$,

$$
\int_{I_{j}} \frac{u^{n+1}(x) u^{n}(x)-u^{n}(x) u^{n-1}(x)}{2 \Delta t} d x+\hat{F}_{j+\frac{1}{2}}^{n}-\hat{F}_{j-\frac{1}{2}}^{n} \leq 0 .
$$

\section{BIBLIOGRAPHY}

1. R. Biswas, K. Devine, and J. Flaherty, Parallel, adaptive finite element methods for conservation laws, Appl. Numer. Math. (to appear).

2. F. Bouchut, C. Bourdarias, and B. Perthame, Un exemple de methode MUSCL satisfaisant toutes les inégalités d'entropie numériques, preprint.

3. B. Cockburn and C.-W. Shu, TVB Runge-Kutta local projection discontinuous Galerkin finite element method for conservation laws II: general framework, Math. Comp. 52 (1989), 411435.

4. B. Cockburn, S. Hou, and C.-W. Shu, The Runge-Kutta local projection discontinuous Galerkin finite element method for conservation laws IV: the multidimensional case, Math. Comp. 54 (1990), 545-581.

5. B. Cockburn, F. Coquel, and P. Le Floch, An error estimate for finite volume methods for multidimensional conservation laws, Math. Comp. (to appear).

6. B. Cockburn and P. Gremaud, An error estimate for finite element methods for scalar conservation laws, preprint. 
7. F. Coquel and P. Le Floch, Convergence of finite difference schemes for conservation laws in several space dimensions: the corrected antidiffusive flux approach, Math. Comp. 57 (1991), 169-210.

8. P. Lions and P. Souganidis, Convergence of MUSCL type methods for scalar conservation laws, C. R. Acad. Sci. Paris 311 (1990), 259-264.

9. C. Johnson, A. Szepessy, and P. Hansbo, On the convergence of shock-capturing streamline diffusion finite element methods for hyperbolic conservation laws, Math. Comp. 54 (1990), 107-129.

10. H. Nessyahu and E. Tadmor, Non-oscillatory central differencing for hyperbolic conservation laws, J. Comput. Phys. 87 (1990), 408-463.

11. S. Osher, Riemann solvers, the entropy condition, and difference approximations, SIAM J. Numer. Anal. 21 (1984), 217-235.

12. Convergence of generalized MUSCL schemes, SIAM J. Numer. Anal. 22 (1985), 947-961.

13. S. Osher and E. Tadmor, On the convergence of difference approximations to scalar conservation laws, Math. Comp. 50 (1988), 19-51.

14. H. Yang, Extremum tracking and convergence of semi-discrete TVD schemes, preprint.

Division of Applied Mathematics, Brown University, Providence, Rhode Island 02912

E-mail address, G. Jiang: gs jecfm.brown .edu

E-mail address, C.-W. Shu: shu@cfm.brown.edu 